\title{
Sound Radiation by Vibrating Building Partitions in Terms of Acceptable Vibration Values
}

\author{
A. SzeląG ${ }^{a}$, K. StypuŁA ${ }^{b}$ And T. KAmisiński ${ }^{a, *}$ \\ ${ }^{a}$ AGH University of Science and Technology, Faculty of Mechanical Engineering and Robotics \\ Department of Mechanics and Vibroacoustics, Al. A. Mickiewicza 30, 30-059 Krakow, Poland \\ ${ }^{b}$ Tadeusz Kościuszko Cracow University of Technology, Civil Engineering Faculty, Institute of Structural Mechanics \\ ul. Warszawska 24, 31-155 Kraków, Poland
}

\begin{abstract}
The subject of this study is an analysis of the phenomenon of sound radiation generated by vibrating building partitions in terms of acceptable vibration values set out in $\mathrm{PN}-88 / \mathrm{B}-02171$ : Evaluation of the vibration impact on people in buildings. The aim of the research was to determine conditions when vibration limits, determined as those providing the required human comfort according to the standard, do not meet the requirements of PN-87/B02151/02 describing sound level limits in rooms. In order to carry out research showing the phenomenon, various aspects of sound radiation by vibrating building partitions were analyzed theoretically. It was assumed that the vibration velocity of elements did not exceed the limits specified in the standard. Calculations were carried out, in particular, for variants with variable thickness or surface of vibrating partition as well as various types of the construction material. Furthermore, the minimum vibration duration was determined for which the values of noise exceeded the sound level limits.
\end{abstract}

DOI: 10.12693/APhysPolA.125.A-122

PACS: 43.20.Tb, 34.50.Ez, 43.40.-r, 43.40.+s

\section{Introduction}

In the course of typical activities carried out in buildings, the occupants are exposed to vibrations, including those transmitted by the ground, generated by means of transport and operation of mechanical devices, as well as vibrations related to presence of people occupying the building and performing various activities following from the building's functional program. These vibrations may have destructive effect on structure of the building and be bothersome for occupants. They can also contribute to an increase of noise level in rooms. In fact, any partition delimiting a room can be considered a separate source emitting acoustic energy to its interior. Any partition excited to vibrations radiates acoustic power in proportion to its surface vibration velocity and the radiation efficiency factor. It is therefore possible to determine the acoustic pressure level in a receiving room based on parameters of vibrations to which partitions delimiting the room are excited.

Foundations for assessment of the effect of vibrations on humans are given in standard PN-88/B-02171 [1]. The norm determines permissible values of mechanical vibrations that are expected to guarantee a necessary level of comfort for people present in rooms of building structures. On the other hand, to determine permissible sound level values in rooms designed for accommodating humans, standard PN-87/B-02151/02 [2] is used. This paper presents results of analysis concerning radiation of

*corresponding author; e-mail: kamisins@agh.edu.pl building partitions delimiting certain rooms under the assumption that the vibration velocities meet requirements concerning permissible values provided by the applicable standard [1]. The aim of the study was to determine under which conditions the permissible parameters of mechanical vibrations that are supposed to guarantee the required comfort for people present in a building, result in failure to meet the requirements of standard [2] imposing thresholds for permissible sound levels in rooms. As the variables defining the problem, the following were selected: plate dimensions and material parameters; constraint type; direction of exiting forces; duration and time of the day of occurrence of the vibrations; and the frequency spectrum of the latter. All the obtained results were compared to calculations carried out for the reinforced concrete partition analyzed in section 3 of this paper. As the permissible A-weighted sound levels in the room, the values of $30 \mathrm{~dB}$ in the night and $40 \mathrm{~dB}$ during the daytime were adopted (living rooms in dwelling houses) [1].

\section{Basic theory}

For the purpose of this paper, a building partition is represented by a rectangular thin plate. Such structures are easy in modeling, and the theory of vibrations of plates is a well-developed branch of mechanics. Moreover, more complex structures can be modeled as systems composed of many individual plate elements. Vibrations of plates can be analyzed from the point of view of both structural modes and the acoustic radiation generated by the structure closely related to its vibration modes. However, the radiating modes, contrary to structural ones, radiate independently from each other. When a flat structure of plate type is subjected to mechanical 
excitation, it starts to vibrate. For the purpose of the present study, rectangular plates with dimensions $l_{x}$ and $l_{y}$ (measured along axes $x$ and $y$, respectively) clamped along all the edges are discussed, whereas the displacement in the direction of $z$ axis, induced mainly by occurrence of the bending wave and being a function of time $t$ in general, is considered to be described by means of the variable $w(x, y, t)$. Despite numerous wave motion types existing in solid structures, bending waves have the strongest effect on displacement along $z$ axis, and thus also on acoustic radiation. The assumptions adopted in order to formulate the equation of transverse motion of a plate include: homogeneity, isotropy, absence of internal forces and damping in the plate, plate thickness being much smaller that the other dimensions, and free propagation of waves. In the case of low frequencies considered herein, these assumptions are well-grounded. The equation of natural vibrations of a plate with the material density $\rho\left(\mathrm{kg} / \mathrm{m}^{3}\right)$ and thickness $h(\mathrm{~m})$ takes the following form[3]:

$$
D \nabla^{4} w(x, y, t)+\rho h \frac{\partial^{2} w(x, y, t)}{\partial t^{2}}=0,
$$

where $D=\frac{E h^{3}}{12\left(1-v^{2}\right)}$ is the bending stiffness of the plate, $E$ is the Young's modulus, and $\nu$ is the Poisson's ratio of the plate material.

The above Eq.(2.1) applies to an unlimited plane. Moreover, the effect of internal damping existing in the structure is neglected. The bending wave can therefore propagate freely starting from the point of excitation. Any actual plate is limited in space, and the waves propagating in it are subjected to reflection, diffraction, and refraction. As a result of the reflection phenomena, vibration amplitudes are amplified for certain frequencies, as the superposing reflected waves form standing waves known also as the wave modes. Such plate vibrates freely only at resonant frequencies.

The next step consists in determination of acoustic power radiated from a vibrating partition [3]. To this end, the structure should be considered a matrix of point sources and a limiting frequency should be determined above which the element will radiate the sound energy. In case of excitation by means of an acoustic wave, such limit arises when the bending wave length in the plate equals the incident sound wave velocity component parallel to the plate surface. In the case of a plate with finite dimensions, a portion of energy can be radiated also below the coincidence frequency, as on the partition edge there is no complete compensation of positive and negative vibration amplitudes. It is therefore practical to use the sound radiation factor $\sigma$ describing efficiency of the acoustic energy radiation process. The acoustic power radiated from a vibrating partition can be determined on the grounds of the following formula:

$$
W=\left\langle\tilde{v}^{2}\right\rangle \rho_{0} c_{0} S \sigma
$$

where $\left\langle\tilde{v}^{2}\right\rangle$ is the time- and space-averaged square of velocity of vibrations of the partition, $\sigma$ is the radiation efficiency factor, $\rho_{0}$ is the air density $\left(\mathrm{kg} / \mathrm{m}^{3}\right), c_{0}$ is the sound velocity in air $(\mathrm{m} / \mathrm{s})$, and $S$ is the vibrating surface area $\left(\mathrm{m}^{2}\right)$.

Finally, the sound level induced in the room by a vibrating partition was determined as

$$
L_{r e c}=10 \log \frac{W}{W_{0}}-10 \log \frac{\{A\}}{4},
$$

where $W$ is the acoustic power radiated out of the vibrating partition $(\mathrm{W}), W_{0}=10^{-12} \mathrm{~W}$ is the sound power reference level, and $A$ is the numerical value of the acoustic absorption of the room (in $\mathrm{m}^{2}$ ).

\section{Methodology}

In order to check whether vibrations of the room do not exceed permissible values determined in $\mathrm{PN}-88$ /B-02171 standard, vibration velocity root mean square values are measured in rooms occupied by people, typically in the center of the ceiling span. On the other hand, to determine the sound power radiated by a vibrating partition, it is necessary to determine the mean square value of vibration velocity for the whole plate surface. To this end, a discrete model of the vibrating partition has been developed and the analysis of vibrations was carried out by means of the finite element method. The model of the partition was created using ANSYS software. Further, the model reflected the plate-shaped type of the element clamped on all edges. A reinforced concrete plate was analyzed with dimensions $3 \mathrm{~m} \times 5 \mathrm{~m} \times 0.2 \mathrm{~m}$. The initial excitation was represented by an appropriate vibration velocity of a point located in the plate center. Velocity values were selected according to the standard [1] (maximum permissible vibration velocities) for determined frequencies. With these assumptions, the modal analysis was carried out aimed at determination of vibrating modes of the partition (Fig. 1) as well as the harmonic analysis the purpose of which was determination of the mean square value of vibration velocity for the plate surface.
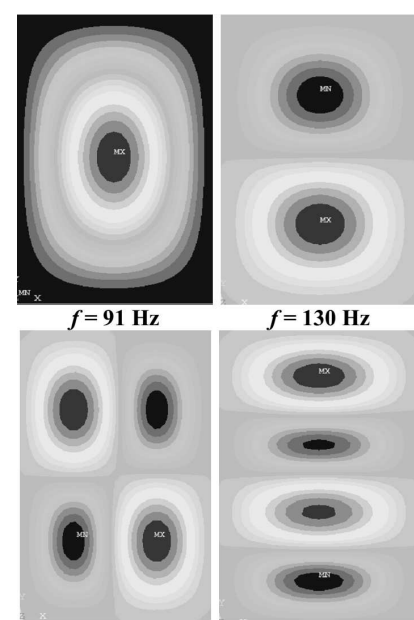

$f=259 \mathrm{~Hz}$

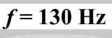

$f=288 \mathrm{~Hz}$

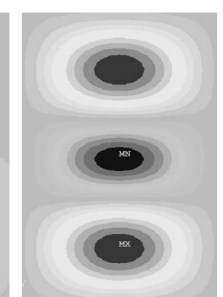

$f=197 \mathrm{~Hz}$
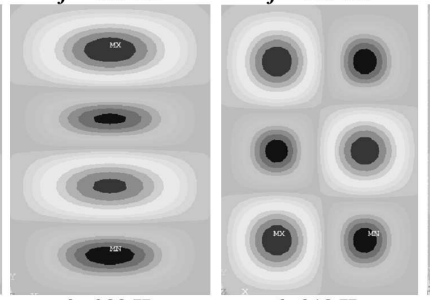

$f=318 \mathrm{~Hz}$

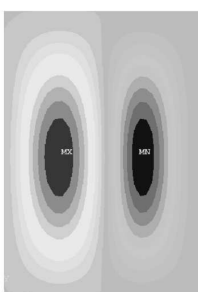

$f=224 \mathrm{~Hz}$

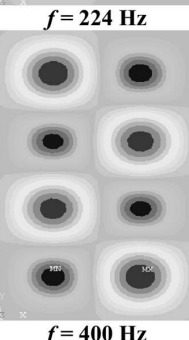

Fig. 1. Vibration modes of a reinforced concrete plate $\left(\rho=2500 \mathrm{~kg} / \mathrm{m}^{3}, E=30 \mathrm{GPa}, v=0.2\right)$ clamped along its edges with dimensions $3 \mathrm{~m} \times 5 \mathrm{~m} \times 0.2 \mathrm{~m}$ listed in the order of increasing natural frequencies. 
Next, the sound radiation efficiency factor characterizing the partitions was determined as a function of vibration frequency. In bands above the coincidence frequency, values of the radiation factor were determined according to the formula [4]

$$
\sigma=\frac{1}{\sqrt{1-\frac{f_{c}}{f}}},
$$

where $f_{c}$ is the partition's coincidence frequency and $f$ is the analyzed vibration frequency. In this frequency range, the sound radiation efficiency value does not depend either on partition geometry or its internal damping. Efficiency of the sound radiation process is affected only by the coincidence frequency value that can be determined from the formula

$$
f_{c}=\frac{c_{0}^{2}}{1,8 c_{L} h},
$$

where $c_{L}$ is the velocity of longitudinal wave in the plate $(\mathrm{m} / \mathrm{s}), h$ is the plate thickness $(\mathrm{m})$, and $c_{0}$ is the velocity of sound in air $(\mathrm{m} / \mathrm{s})$.

Various formulas allowing to calculate values of the radiation efficiency factor for the range below the coincidence frequency were published by a number of authors. For the purpose of this study, the following formulas proposed by Cremer $[4,5]$ have been adopted:

$$
\begin{aligned}
\sigma & =\frac{1}{\pi^{2}} \frac{U}{S} \lambda_{c} \sqrt{\frac{f}{f_{c}}} \text { for } f \ll f_{c} \\
\sigma & =0.45 \sqrt{\frac{U}{\lambda_{c}}} \text { for } f=f_{c},
\end{aligned}
$$

where $f_{c}$ is the partition's coincidence frequency, $f$ is the analyzed vibration frequency, $U$ is the plate perimeter, $S$ is the plate area and $\lambda_{c}$ is the wavelength of the coincidence frequency.

The next step consisted in calculation of the sound level in the receiving room according to formulae (2.2) and (2.3). For the purpose of calculations it has been assumed that vibrations are stationary in $x / y$ direction (a human in lying position during the nighttime), and therefore the value of coefficient $n=1.4$ has been adopted from Table 5 in standard [1]. Acoustic absorption of the room was selected according to standard [2] for living rooms $\left(A=10 \mathrm{~m}^{2}\right)$. Acoustic power radiated from two partitions (ceiling and floor) was taken into account in the analysis. Results of calculations are presented in Table I.

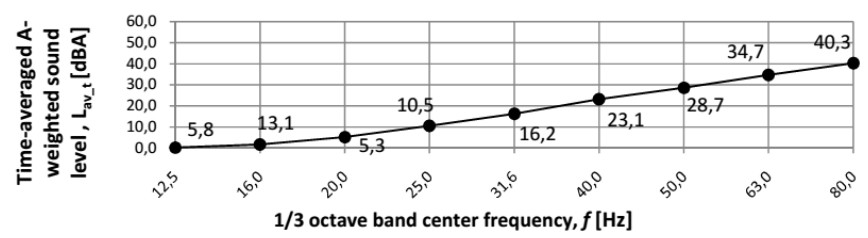

Fig. 2. Time-averaged A-weighted sound level in the room as a function of partition vibration frequency.

The next step of the calculation algorithm consisted in determination of A-weighted sound level in the receiving
TABLE I

Algorithm used to determine the sound level in the receiving room.

\begin{tabular}{l|c|c|c|c|c}
\hline \hline$f$ & $\left\langle v^{2}\right\rangle$ & $\sigma$ & \multicolumn{2}{|c|}{$10 \log (\ldots)[\mathrm{dB}]$} & $L_{\text {rec }}$ \\
\hline$[\mathrm{Hz}]$ & {$[\mathrm{m} / \mathrm{s}]$} & {$[-]$} & $\left(W / W_{0}\right)$ & $(\{A\} / 4)$ & {$[\mathrm{dB}]$} \\
\hline 12.5 & $1.14 \times 10^{-8}$ & 0.1472 & 73.17 & 3.98 & 69.2 \\
16.0 & $1.15 \times 10^{-8}$ & 0.1665 & 73.74 & 3.98 & 69.8 \\
20.0 & $1.16 \times 10^{-8}$ & 0.1862 & 74.27 & 3.98 & 70.3 \\
25.0 & $1.18 \times 10^{-8}$ & 0.2082 & 74.83 & 3.98 & 70.8 \\
31.6 & $1.21 \times 10^{-8}$ & 0.2340 & 75.44 & 3.98 & 71.5 \\
40.0 & $1.27 \times 10^{-8}$ & 0.3753 & 77.70 & 3.98 & 73.7 \\
50.0 & $1.37 \times 10^{-8}$ & 0.4546 & 78.87 & 3.98 & 74.9 \\
63.0 & $1.57 \times 10^{-8}$ & 0.6328 & 80.89 & 3.98 & 76.9 \\
80.0 & $2.04 \times 10^{-8}$ & 0.7475 & 82.75 & 3.98 & 78.8
\end{tabular}

room averaged over time (Fig. 2). The whole evaluation period $t_{c}$ was adopted as equaling 8 hours (night-time, 10 p.m. to 6 a.m.). It has been also assumed that the total time for which the vibrations occurred was 12 minutes.

\section{Results}

The first of the comparative analyses carried out in the framework of the present study pertained to radiation of sound generated by plates with different face dimensions and thicknesses. Figure 3 presents sound level calculation results for vibrating structures with face dimensions $1 \mathrm{~m} \times 2 \mathrm{~m}, 3 \mathrm{~m} \times 5 \mathrm{~m}$, and $5 \mathrm{~m} \times 6 \mathrm{~m}$, while Fig. 4 show results obtained for plates 10,15 , and $20 \mathrm{~cm}$ thick.

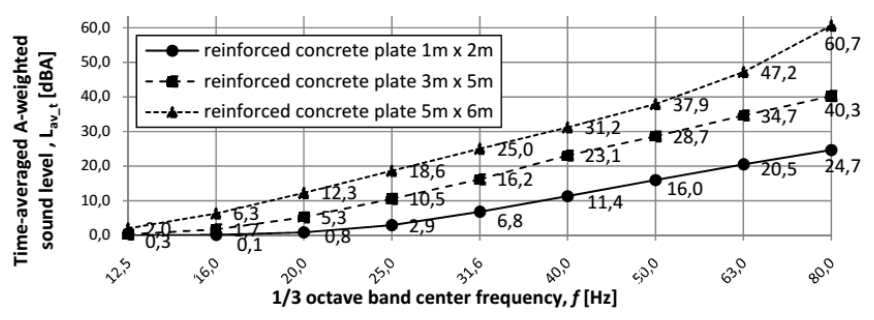

Fig. 3. Time-averaged A-weighted sound levels in the receiving room induced by vibrating plates with dimensions $1 \mathrm{~m} \times 2 \mathrm{~m}, 3 \mathrm{~m} \times 5 \mathrm{~m}$, and $5 \mathrm{~m} \times 6 \mathrm{~m}$.

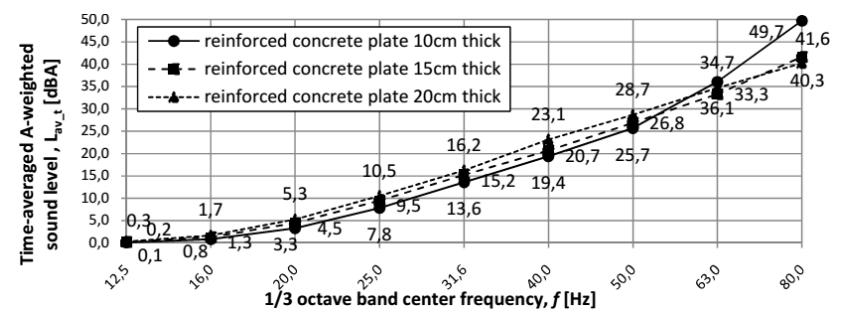

Fig. 4. Time-averaged A-weighted sound levels in the receiving room induced by vibrating plates 10,15 , and $20 \mathrm{~cm}$ thick.

When analyzing Fig. 3 it can be noted that the noiserelated values provided by applicable standards were not exceeded only in case of the plate with dimensions $1 \mathrm{~m} \times 2 \mathrm{~m}$ (the maximum sound level was $24.7 \mathrm{~dB}$ for frequency $80 \mathrm{~Hz}$ ). Together with increasing dimensions of 
the analyzed partition, deviations from permissible values increase accordingly by even as much as $100 \%$ (plate $5 \mathrm{~m} \times 6 \mathrm{~m}$, frequency $80 \mathrm{~Hz}$ ). The cases of maximum excess above the applicable standard noise thresholds have been obviously met for higher frequencies in view of the characteristics of sound perception by humans which is reflected in properties of the A-weighting curve. Nevertheless, for the $5 \mathrm{~m} \times 6 \mathrm{~m}$ plate, the permissible sound level in the night-time is exceeded already for the frequency as low as $40 \mathrm{~Hz}$. It is also worth noting that the maximum calculated sound level was $60.7 \mathrm{~dB}$. This twofold excess above the value provided by the standard may suggest that the partition vibration velocities even much less than the limiting value can result in occurrence of excessive noise.

The second of the analyzed graphs (Fig. 4) depict the effect of partition thickness on sound level in a room. For lower frequencies, differences between the characteristics are small, and the minimum sound level values are induced by the thinnest plate. This is directly related with the sound radiation factor values that for the $10-\mathrm{cm}$ thick element are lowest (Fig. 5). In higher frequency bands, i.e. those centered at $63 \mathrm{~Hz}$ and $80 \mathrm{~Hz}$, the noise threshold values provided by the standards are exceeded for all plate types. However, here the maximum sound level value was obtained for the thinnest structure, despite the lowest radiation factor value. The cause was a very large increase of vibration velocity of the plate in view of its small rigidity, and the resulting occurrence of resonance frequencies in the analyzed frequency bands (Table II).

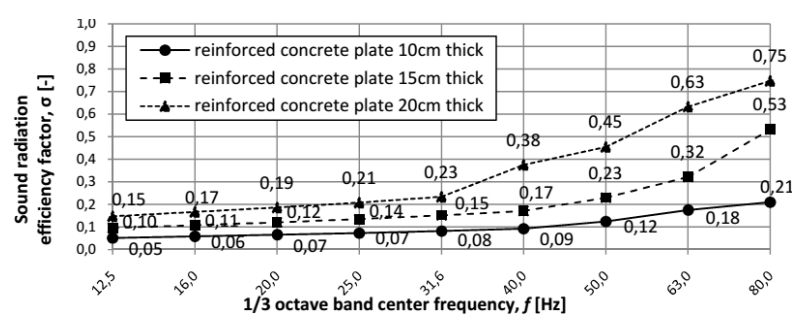

Fig. 5. The sound radiation efficiency factor for vibrating plates 10,15 , and $20 \mathrm{~cm}$ thick.

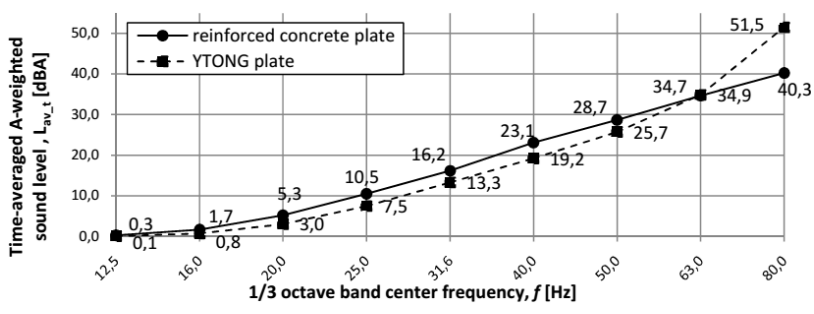

Fig. 6. The time-averaged A-weighted sound levels in the reception room generated by vibrations by reinforced concrete and YTONG plates.

Another comparison concerns vibrating reinforced concrete (RC) floors opposed to floors made of YTONG slabs (reinforced cellular concrete). The change involves there-

\section{TABLE II}

Five successive frequencies of natural vibrations $f_{s}$ for plates 10, 15, and $20 \mathrm{~cm}$ thick and their coincidence frequencies.

\begin{tabular}{c|c|c|c|c|c|c}
\hline \hline$d[\mathrm{~cm}]$ & \multicolumn{5}{|c|}{$f_{s}[\mathrm{~Hz}]$} & $f_{c}[\mathrm{~Hz}]$ \\
\cline { 2 - 6 } & $\# 1$ & $\# 2$ & $\# 3$ & $\# 4$ & $\# 5$ & Eq. (3.1) \\
\hline 10 & 47 & 67 & 102 & 117 & 135 & 185.4 \\
\hline 15 & 69 & 99 & 151 & 172 & 199 & 123.6 \\
20 & 91 & 130 & 197 & 224 & 259 & 92.7
\end{tabular}

fore material parameters of the partitions, and in particular their mass density $\left(\rho_{R C}=2500 \mathrm{~kg} / \mathrm{m}^{3}, \rho_{Y \text { tong }}=\right.$ $\left.550 \mathrm{~kg} / \mathrm{m}^{3}\right)$ and Young's modulus $\left(E_{R C}=30 \mathrm{GPa}\right.$, $\left.E_{Y \text { tong }}=2 \mathrm{GPa}\right)$. When analyzing results of the study (Fig. 6) it can be seen that in lower frequency bands, more harmful were vibrations of reinforced concrete partition in view of higher levels of the radiated sound. In case of the frequency $80 \mathrm{~Hz}$, a rapid increase of the emitted noise has been observed. The reason was occurrence of the resonance frequency equaling $72 \mathrm{~Hz}$ in the analyzed frequency band, caused by significant decrease of rigidity of the plate.

The next comparison concerned plates with different types of constraints (Fig. 7). The first of the considered plates were clamped along all its edges, the second was clamped along its $3 \mathrm{~m}$ long sides and supported along the $5 \mathrm{~m}$ edges, while the last plate was supported along the whole of its perimeter.

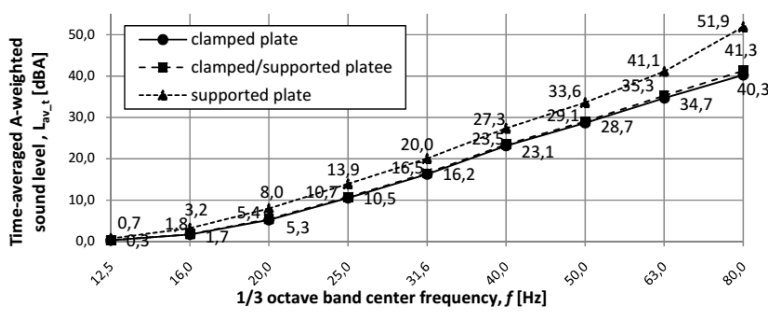

Fig. 7. Time-averaged A-weighted sound levels in the receiving room induced by vibration of plates with different constraints.

Analysis of Fig. 7 leads to the conclusion that the presented characteristics are very similar for plates clamped along either one or all the four of their edges. On the other hand, the sound level increases when the element is simply supported along the whole perimeter. The differences can be as high as $10 \mathrm{~dB}$ which will lead to significant overstepping of the values permitted by standards (by $22 \mathrm{~dB}$ for $80 \mathrm{~Hz}$ frequency band).

So far, only vibrations in $x$ and $y$ directions were considered occurring in the night-time. For the sake of comparison, a situation has been also modeled where the vibrations were received in $z$ direction (standing position) and the analyzed time was the daytime. In such case, the maximum velocity of vibrations was $0.0001 \mathrm{~m} / \mathrm{s}$, and the correction coefficient $n$ was 4 [1]. It can be easily noted that the corrected velocity values are almost identical 
to those in the case analyzed earlier (vibration velocity $0.00029 \mathrm{~m} / \mathrm{s}$, correction coefficient $n=1.4$ ). Also the sound levels in the receiving room are the same. The only change that should be taken into account in the course of the analysis is the permissible noise level that for the daytime equals $40 \mathrm{~dB}$. A review of the whole of characteristics presented above leads to the conclusion that under the adopted assumptions, the noise-related standard thresholds were exceeded mainly in the band centered at frequency of $80 \mathrm{~Hz}$.

In the next step of the analysis, different total periods of times were assumed for which partition vibrations occurred. In the daytime, excitations lasting 15, 30, and 60 min were taken into account (Fig. 8). In the nighttime, vibrations occurring for 6,15 , and $30 \mathrm{~min}$ were assumed (Fig. 9).

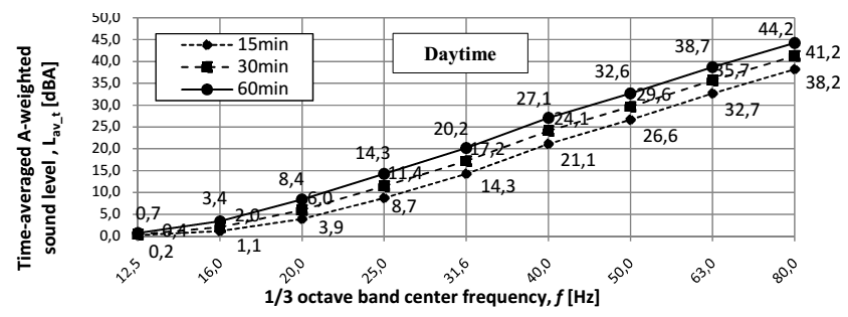

Fig. 8. Time-averaged A-weighted sound levels in the receiving room induced by vibrations of building partitions lasting for different periods of time (daytime).

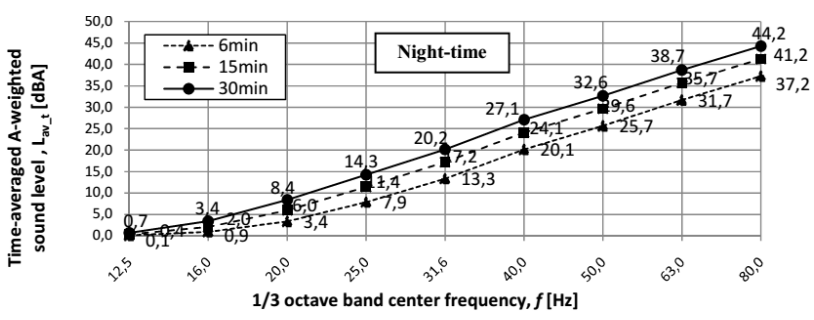

Fig. 9. Time-averaged A-weighted sound levels in the receiving room induced by vibrations of building partitions lasting for different periods of time (night-time).

Analyzing the above graphs it can be noted that in the case of vibrations occurring in the daytime, the permissible noise thresholds provided by the standards have been exceeded only for the frequency $80 \mathrm{~Hz}$ and excitation lasting for 30 or $60 \mathrm{~min}$. The situation in the night-time turns out to be much worse. Here, vibrations lasting as short as only 6 min result in exceeding permissible sound levels in $63 \mathrm{~Hz}$ and $80 \mathrm{~Hz}$ frequency bands. On the other hand, vibration lasting $30 \mathrm{~min}$ induces excessive noise also for the frequency $50 \mathrm{~Hz}$, and in the $80 \mathrm{~Hz}$ band, the limit provided by the standard is exceeded by as much as $14 \mathrm{~dB}$.

\section{Conclusions and further work}

On the grounds of the research work carried out to date it can be concluded that vibrations of building partitions delimiting a receiving room, determined as permissible ones as per standard [1], may results in violation of noiserelated standards. Maximum oversteppings are observed for higher frequencies from the analyzed range in view of perception characteristics typical for humans reflected in A-type sound level weighting curve. With increasing dimensions of the partition, the sound level induced in the room by its vibrations also increases, resulting in significant exceeding the noise control standard limits. The sound level value depends also on thickness of the vibrating building partitions delimiting the room. Less noise is generated by thinner plates that in general have lower radiation efficiency factors; however, this property can be easily subjected to disturbance in connection with occurrence of a resonance frequency in the analyzed band. Moreover, specific material parameters of the element, i.e. its low rigidity or high mass density, result in decrease of the resonance frequency value which, as a consequence, appears in the analyzed frequency band and induces significant increase of acoustic power generated from the vibrating partition. The acoustic power radiated from a vibrating plate depends also strongly on the type of constraints representing different mounting methods and increases with releasing consecutive degrees of freedom. In case of a vibrating partition radiating acoustic energy into a room, surpassing of applicable noise control standard thresholds could be observed even for excitations lasting just a few minutes.

The problems raised in this paper constitute a beginning of an extensive research project concerning propagation of the material sound and radiation of acoustic energy by various structures. Exploration of phenomena related to transmission of material sound in various structures and acoustic radiation induced by them will allow to identify and minimize the resulting noise sources as early as in the construction design stage. Further plans include extension of the model onto entire building structures with different design solutions and technologies taken into account. Moreover, it is planned that the analysis will take into account kinematic excitations in the form of time-dependent waveforms representing vibrations of actual building structures obtained from measurements.

\section{References}

[1] PN-88/B-02171: Evaluation of vibrations influence onpeople in buildings.

[2] PN-87/B-02151/02: Acoustics in building. Noise protection facilities in buildings. The permissible sound level values on the premises.

[3] L. Cremer, M. Heckl, Structure-borne sound. Structural Vibrations and Sound Radiation at Audio Frequencies, Springer-Verlag, Berlin 1988.

[4] A. Osama, B. Hassan, Building Acoustics and Vibration. Theory and Practice, World Scientific, Singapore 2009.

[5] A. Polaczek, J. Rubacha, T. Kamisiński, XVI Conference on Acoustic and Biomedical Engineering, Kraków-Zakopane 2009, p. 151. 